\title{
A Robust Identification of the Protein Standard Bands in Two-Dimensional Electrophoresis Gel Images
}

\author{
Artūras Serackis* (Professor, Vilnius Gediminas Technical University), \\ Dalius Matuzevičius (Professor, Vilnius Gediminas Technical University), \\ Dalius Navakauskas (Professor, Vilnius Gediminas Technical University), \\ Eldar Šabanovič (Junior Researcher, Vilnius Gediminas Technical University), \\ Andrius Katkevičius (Associate Professor, Vilnius Gediminas Technical University), \\ Darius Plonis (Associate Professor, Vilnius Gediminas Technical University)
}

\begin{abstract}
The aim of the investigation presented in this paper was to develop a software-based assistant for the protein analysis workflow. The prior characterization of the unknown protein in two-dimensional electrophoresis gel images is performed according to the molecular weight and isoelectric point of each protein spot estimated from the gel image before further sequence analysis by mass spectrometry. The paper presents a method for automatic and robust identification of the protein standard band in a two-dimensional gel image. In addition, the method introduces the identification of the positions of the markers, prepared by using pre-selected proteins with known molecular mass. The robustness of the method was achieved by using special validation rules in the proposed original algorithms. In addition, a self-organizing map-based decision support algorithm is proposed, which takes Gabor coefficients as image features and searches for the differences in preselected vertical image bars. The experimental investigation proved the good performance of the new algorithms included into the proposed method. The detection of the protein standard markers works without modification of algorithm parameters on twodimensional gel images obtained by using different staining and destaining procedures, which results in different average levels of intensity in the images.
\end{abstract}

Keywords - Biomedical imaging; Image analysis; Molecular biomarkers; proteins; quantization.

\section{INTRODUCTION}

Two-dimensional electrophoresis (2DE), which was introduced more than 40 years ago [1], is still the most widely used approach in top-down proteomic studies for all types of biological models. It plays an important role for the identification of protein sequences and further functions within the cell.

Due to the low 2D gel electrophoresis image reproducibility and the complexity of 2DE maps, it is necessary to develop additional techniques for 2DE image acquisition and analysis that are effective and reliable in the comparison of $2 \mathrm{DE}$ images and 2D mapping classification. To achieve this, it is necessary to develop advanced methods for 2DE image acquisition and data analysis [2].

The aim of the automation in 2DE gel image analysis is to increase the amount of useful information gathered from a single gel and all the corresponding gels in the dataset under investigation [3]-[8]. The estimation of the protein molecular weights (MW) in the gel is performed by processing the gel with the desired stain and then destaining it in order to visualize the protein bands. The relative migrating distance $\left(R_{\mathrm{f}}\right)$ of the protein standards and of the unknown protein is defined as the mobility of the protein divided by the mobility of the ion front. The molecular weight estimate of the unknown protein band is obtained by interpolating the value from the $\log \left(m_{\mathrm{MW}}\right)$ vs. $R_{\mathrm{f}}$ graph. This graph should be linear for most proteins.

The markers on the 2DE gel (Fig. 1) are introduced by using protein standards with known $m_{\mathrm{MW}}$ values, e.g. $m_{\mathrm{MW}}=\{250 ; 130 ; 100 ; 70 ; 55 ; 35 ; 27 ; 15 ; 10\}$. The MW of an unknown protein band is determined from the graph, that uses the $R_{\mathrm{f}}$ values of the marker. These $R_{\mathrm{f}}$ values are usually determined graphically [9]:

Step 1. A ruler measures the migration distance from the top of the resolving gel to the dye front.

Step 2. The value is calculated for each band in the standards with known MW values.

Step 3. The measured points are approximated by an equation $y=k x+b$. Here $y=\log \left(m_{\mathrm{MW}}\right)$ and $x=R_{\mathrm{f}}$.

Step 4. Step 2 is repeated for each unknown protein band in the sample.

Step 5. The MW of the unknown protein is estimated by solving the line equation for $y$.

The solution proposed in this paper accelerates the procedure of MW estimation for an unknown protein band. The identification of the protein standards marker positions is performed automatically by applying a sophisticated image analysis algorithm to the 2DE gel image.

\footnotetext{
* Corresponding author.

E-mail: arturas.serackis@vgtu.lt
} 


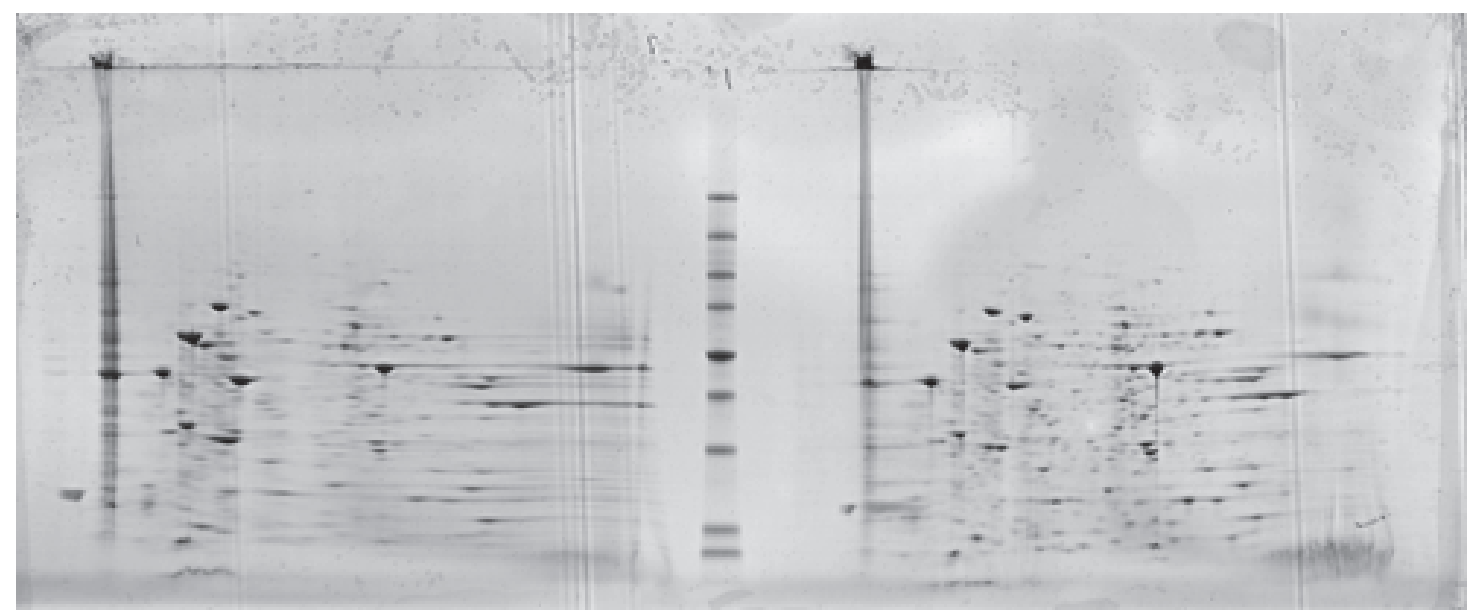

Fig. 1. Example of two-dimensional electrophoresis gel image, selected for protein MW marker detection (the protein standards are located in the middle of the image).

\section{MATERIALS AND METHODS}

\section{A. An Overview of the Approach}

This paper presents a four-stage image analysis method for the detection of MW marker positions in the 2DE gel.

Stage 1 . Read the 2DE gel image and apply a modified filter for estimating the image phase symmetry.

Stage 2. Apply an asymmetric Gaussian-smoothing filter.

Stage 3. Accumulate the image pixel intensities in each column of the image matrix.

Stage 4. Analysis of the vertical regions of the image.

The following subsections present an explanation of each stage in detail.

\section{B. Image Phase Symmetry Estimation}

During this initial stage of the proposed method, the horizontally structured elements are emphasized in the image. The known MW protein spots received from the standard appear as spots that are symmetrical in relation to the horizontal axis of the image. In order to emphasize the horizontal symmetry for each object in the image, a modified image phase symmetry estimation filter is applied. The filter is based on the one proposed in [10], [11]. This filter does not require prior segmentation of the image to calculate the symmetry estimate for each image object individually.

A modification of the initial author implementation was performed by changing the range of divided frequencies from $[0, \pi]$ to $[-\pi / 8, \pi / 8]$. The frequency range was divided into five equal bands and five oriented filters were used. Such modification highlighted only horizontal symmetry in the image (Fig. 2). As is usual in 2DE gel image analysis [12], the image is inverted before the processing steps are started.

\section{Application of Gaussian Smoothing Filter}

The application of the phase-symmetry filter does not remove noise-related structures in the image. These elements in the image appear during the storage of the gel and its displacement and because of gel's susceptibility to dust [13].
Although a Gaussian filter, which is frequently used in 2DE gel image pre-processing, is not the best solution for protein spot boundary estimation and there are alternative solutions [14], the selection of this filter for the current method is justified by two aspects. There is a possibility to select two different standard deviations for the Gaussian smoothing kernel and form an asymmetrical spatial filter. The horizontal standard deviation of the Gaussian smoothing filter used in our proposed method was selected six times larger than the vertical one. The results of the image smoothing are shown in Fig. 2.

After the smoothing of the image, all the pixel intensities in each column of the image matrix are accumulated. Such horizontal analysis (Fig. 2) is used in the next step of the proposed method in order to divide the image into vertical regions and perform an individual analysis of the vertical distribution of the pixel intensities for each region.

\section{Analysis of Vertical Image Regions}

During this stage, the original six-step algorithm for the identification of the MW marker line is executed.

Step 1. The peaks (local minima and local maxima) are found in the calculated horizontal distribution of intensities. As a result of applying an asymmetrical Gaussian filter, the horizontal distribution of the accumulated pixel intensities has minimum amount of noisy peaks. Therefore, in the next step of the algorithm, the image can be divided into vertical bars (Fig. 3) with one accumulated intensity maximum per bar and a width defined by two local minima found to the left and to the right of the maximum.

Step 2. The difference in the total intensities between each local maximum point and the neighbouring local minimum points is estimated. The local minima to the left and to the right of the maximum point may have different magnitudes. Therefore, the average of the local minima intensities is used to calculate the difference between the local maxima and local minima levels. 


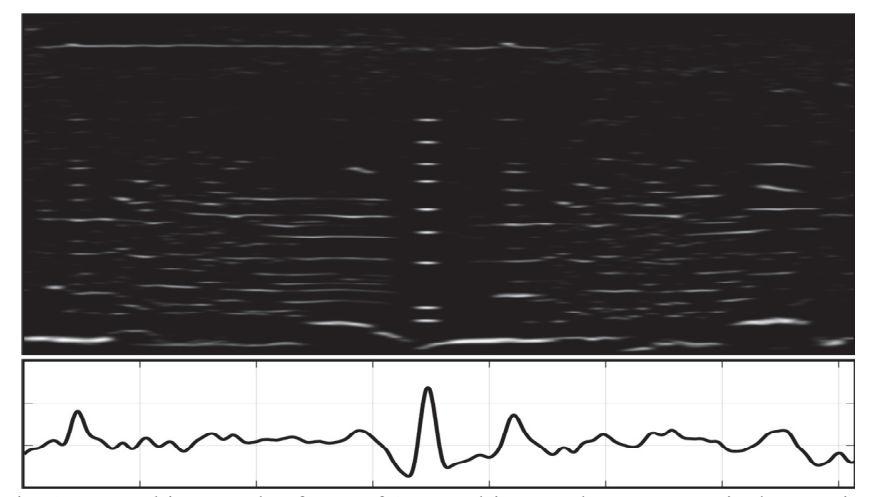

Fig. 2. Smoothing result of one of 2DE gel images by asymmetrical Gaussian filter with illustrated graph of pixel intensity accumulation result for each image matrix column.

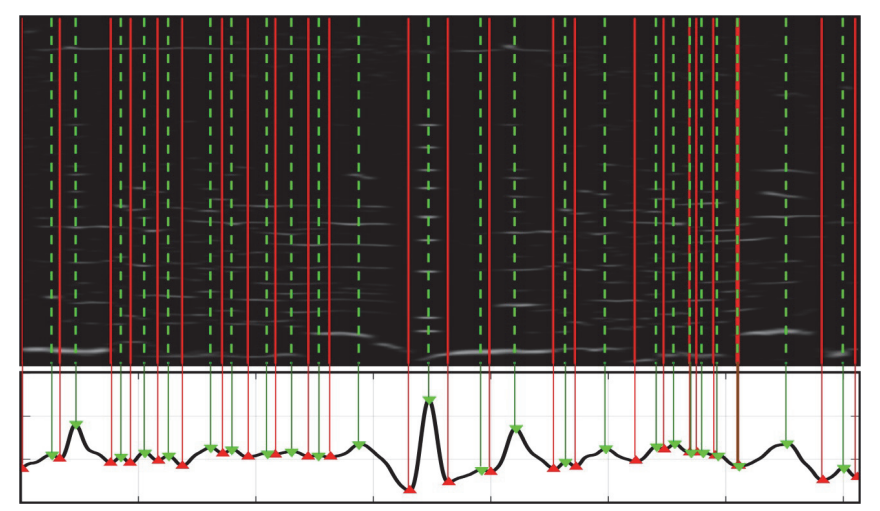

Fig. 3. Result of division into vertical bars of one of 2DE gel images according to the location of local minima and local maxima in the horizontal distribution of intensity values.

The estimation of the difference between the local minima and local maxima levels in each vertical bar of the image is based on the assumption that the MW markers received from the protein standard (usually, they have similar intensities) will be vertically aligned and lead to higher level differences compared to the unknown, unaligned protein spots. Such an assumption is true for many 2DE gel images; however, this feature is insufficient for robust detection of an image bar with MW protein markers in it. It is seen in Figure 1 that the $2 \mathrm{DE}$ gel image may have several vertical bars with higher pixel intensities aligned vertically. Therefore, an additional analysis of the vertical distribution of the pixel intensities in each bar is performed in the following steps of the algorithm.

Step 3. The number of local maxima of pixel intensities in each vertical bar is analysed. Not all the image pixel columns are analysed in the vertical bar. The number of image matrix columns is selected in the range, taking $2 / 3$ of the distance between the local maximum and the local minimum to the left, and $2 / 3$ of the distance between the local maximum and the local minimum to the right.

All the peaks found in the vertical distribution of the pixel intensities are compared to the highest peak. Peaks with a magnitude below $30 \%$ of the magnitude of the highest peak are removed from further analysis.

Step 4. All the 2DE gel image vertical bars are sorted according to the magnitude of the peak with the highest intensity. Fifty percent of the image bars with the smaller intensity of the highest peak are removed from further analysis.
Step 5. The pattern of the theoretical marker distribution is prepared and matched to the peak distribution in each vertical bar. A detailed explanation of Step 5 of the proposed algorithm is provided in the following subsection.

Step 6. A check for the presence of several marker bars is performed. 2DE gel images selected for analysis or taken from different databases may have two MW marker bars in them. Therefore, in order to increase the robustness of the proposed algorithm, additional verification of two vertical bars with the lowest root-mean-square (RMS) error is performed. If the RMS error values of two bars do not differ at least 1.5 times, comparison with the bar with the third-lowest RMS error is performed. In order to select the vertical bar as the one with MW markers, each from two should have an estimated RMS error value that is 1.5 times or more lower compared to the third one.

\section{E. Pattern Matching}

The pattern-matching in our proposed algorithm is performed by taking the theoretical (expected) distribution of the protein standard MW markers and comparing it to the distribution of the intensity peaks in each selected image bar. The pattern vector is prepared by taking seven values from $m_{\mathrm{MW}}=\{250 ; 130 ; 100 ; 70 ; 55 ; 35 ; 27 ; 15 ; 10\}$. Vector $[130$; $100 ; 70 ; 55 ; 35 ; 27 ; 15]$ is created by removing the lowest and the highest values from the $m_{\mathrm{MW}}$ set. These seven values are converted into a logarithmic scale and are then normalized to the range from 0 to 1 .

Taking into account that the protein standard has $9 \mathrm{MW}$ markers, each image bar (from the ones selected during Step 4 of the algorithm) is verified to have at least seven peaks in a row with a magnitude exceeding $30 \%$ of the peak with the highest intensity. In addition, all image bars with more than eleven such peaks are eliminated from further patternmatching steps.

In order to prepare the intensity peak distribution in the bar for pattern-matching, a row of the nine highest peaks are taken from the bar and the highest and the lowest two out of these nine are eliminated. The coordinates of the remaining 7 intensity peaks are then normalized to the range $[0 ; 1]$.

A root-mean-square (RMS) value of the differences between the reference marker positions and the normalized peak positions in the image bar is calculated for each bar. Two image bars with the lowest RMS values are compared. If the RMS values differ 1.5-fold, the column with the lowest RMS value is annotated as a column with MW markers (Fig. 4).

\section{F. Protein Isolation and Fractionation in 2DE System}

Acute promyelocytic NB4 cells of the human blood cancer cell line were cultured in RPMI medium (Gibco Invitrogen, Lidingö, Sweden) containing $10 \%$ foetal bovine serum (FBS), $100 \mathrm{U} / \mathrm{ml}$ penicillin, $100 \mu \mathrm{g} / \mathrm{ml}$ streptomycin (Gibco), and $20 \mathrm{mM}$ glutamine at $37^{\circ} \mathrm{C}$ in humidified air and at $5 \% \mathrm{CO}_{2}$. Leukemic cells for granulocytic differentiation were treated with $1 \mu \mathrm{M}$ retinoic acid (ATRA) for $96 \mathrm{~h}$. The retinoic acid was purchased from Sigma Chem. Co. (St. Louis, MO, USA). 


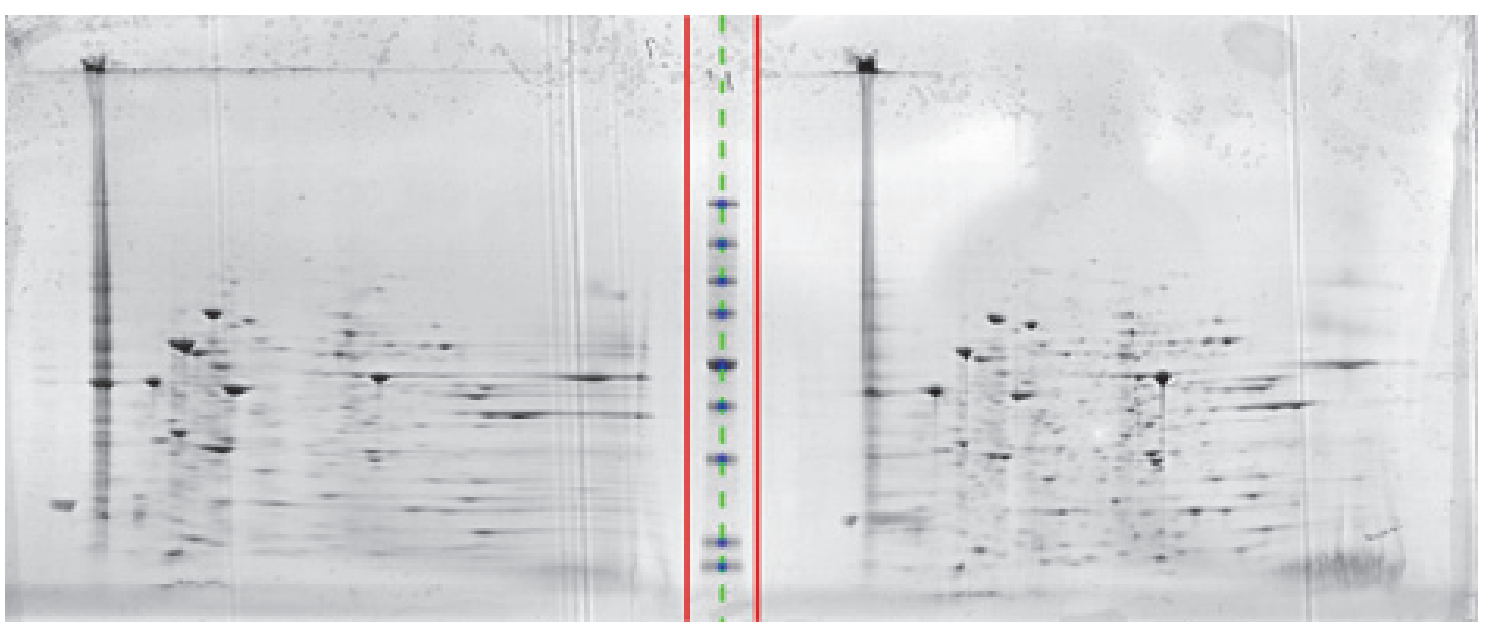

Fig. 4. Illustration of protein standard MW marker identification result on one of the 2DE gel images in the test dataset.

Leukemic cells were induced for granulocytic differentiation by treatment with ATRA for $96 \mathrm{~h}$. Leukemic and differentiated cells were subjected to the isolation of cytoplasmic proteins by using the Nuclear Isolation Kit (Sigma) according to the manufacturer's instructions. An Immobiline DryStrip Kit, pH range 3-10, and Excel Gel SDS, gradient $8 \%$ to $18 \%$, was used for two-dimensional electrophoresis. It was carried out according to the manufacturer's instructions (Immobiline DryStrip Kit for 2D Electrophoresis with Immobiline DryStrip and ExcelGel SDS, Pharmacia Biotech).

The 2DE gels were stained with Coomassie stain for $2 \mathrm{~h}$ or $24 \mathrm{~h}$ and then silver-stained according to the manufacturer's instructions (Thermo Fisher Scientific Inc., Waltham, MA, USA).

\section{EXPERIMENTS AND RESULTS}

All image-processing and decision-making algorithms of the proposed MW marker identification method were implemented and tested in MATLAB environment. A set of 2DE gel images were taken from the biological experiment described in the section "Materials and Methods". Shortly, leukaemia cells (NB4 cell line) were left untreated or treated with differentiation inducer ATRA for 96 hours. Isolated proteins were fractionated in the 2DE system and then visualized by different dyes (Coomassie Brilliant Blue and Silver stain).

For protein characterization it is important to determine the exact molecular weight (MW) and isoelectric point (pI) of each protein spot in 2DE before further sequence analysis by mass spectrometry (Fig. 5). These two characteristics (MW and $\mathrm{pI}$ ), are the most important ones for the description of proteins in 2DE. In order to ensure that the MW marker identification method is robust for $2 \mathrm{DE}$ gel images with different staining techniques, three versions of each gel were prepared. Proteins separated by 2DE can be visualized using different staining procedures. Coomassie staining is simple and compatible with mass spectrometry techniques and up to $36 \mathrm{ng}$ to $47 \mathrm{ng}$ of protein per spot can be detected. Silver staining is more sensitive and up to $0.6 \mathrm{ng}$ to $1.2 \mathrm{ng}$ of protein per spot can be detected. In this study, 2DE gels were stained with Coomassie Brilliant Blue dye for $2 \mathrm{~h}$ and $24 \mathrm{~h}$ to and then stained with Silver dye as described in the section "Materials and Methods".

The experimental investigation performed on the $452 \mathrm{DE}$ gel images selected for testing has shown that the threshold values selected for the algorithms in the proposed method are acceptable because these algorithms did not fail with the test images. However, in order to prove the effectiveness of these values, 2DE gel images taken from different experiments, with different protein standards and different standard positions in the gel, must be tested.

The visual analysis of the 2DE gel images, prepared by different research centres and laboratories, provided in twodimensional polyacrylamide gel electrophoresis database SWISS-2DPAGE or similar databases, has shown the possible variability of the protein spot distributions in the image. However, the MW marker bars present in these images are easily noticed and detected by the human eye. One of the visual features of the MW markers is the similarity of the marker spots in the standard bar.

We propose a self-organizing feature map (SOM) for estimating the similarities in data taken from the vertical bars of the 2DE gel image. We assumed that the distribution of the intensities of the unknown protein spots in the vertical bar might be similar to the distribution of the MW marker intensities. However, the shape of the unknown protein spots will be misaligned and will probably have different shape features that can be visually noticed by a human observer.

The verification is performed in several steps. First, a square image segment is taken around each intensity peak in the image bar (a smoothed image, obtained after Stage 2 of the proposed method, is used). Second, the 2D Gabor wavelet transform [15] is applied to each extracted image square segment. The Gabor wavelet is selected because of the image representation, which is close to the population of V1 simple cells in human vision [16]. 


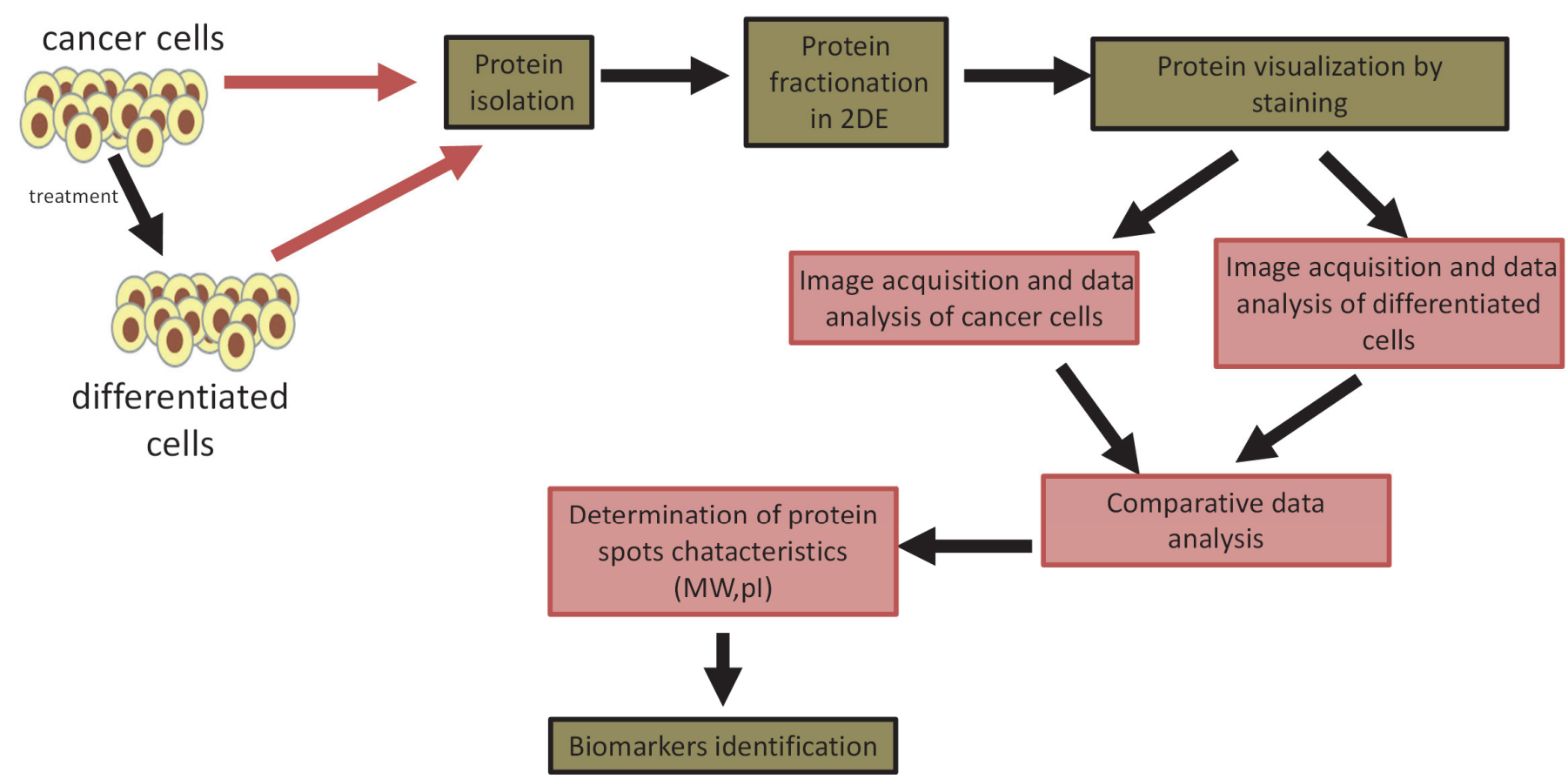

Fig. 5. Result of image bar selection and identification of protein standard MW markers in 2DE gel image.

The Gabor coefficients from all the square image segments in the analysed vertical bar were sent to the $5 \times 5$-SOM-based classificatory. The number of peaks defined for the same class should be at least nine if all MW markers have the same features, based on the Gabor coefficient. The proposed SOM classifier, which is based on the Gabor coefficient, is used as a decision support technique together with the pattern-matching algorithm presented in Subsection E.

\section{DISCUSSION AND CONCLUSION}

In this paper, we propose a method for automatic identification and localization of MW markers in 2DE gel images. The proposed method includes original algorithms for image pre-processing and pattern-matching. The experimental investigation of the method using the selected images that were specially prepared for robustness testing has shown that the several threshold values that were selected for the proposed algorithms are effective enough to detect the image bar with MW markers. However, there is no possibility of testing the robustness of the method on all types of 2DE gel images. Therefore, an additional verification technique, based on the features of human vision and a self-organizing feature map, is proposed. This technique was able to support all the decisions made by a proposed method as the correct ones.

\section{CONFLICT OF INTEREST STATEMENT}

The authors report no conflicts of interest.

\section{ACKNOWLEDGEMENTS}

This study was funded by the Research Council of Lithuania [grant number MIP-083/2015].

\section{REFERENCES}

[1] P. H. O'Farrell, "High Resolution Two-Dimensional Electrophoresis of Proteins," J. Biol. Chem., vol. 250, no. 10, pp. 4007-4021, 1975.

[2] E. Marengo, E. Robotti, and M. Bobba, "2D-PAGE Maps Analysis," Clinical Proteomics, pp. 291-325, 2008. https://doi.org/10.1007/978-1-59745-117-8 16

[3] A. Serackis, D. Matuzevičius, and D. Navakauskas, "2DE Gel Image Preprocessing Using Self-Organising Maps," Photonics Applications in Astronomy, Communications, Industry, and High-Energy Physics Experiments 2010, Jun. 2010. https://doi.org/10.1117/12.871866

[4] G. Treigytè, I. Zaikova, D. Matuzevičius, V. Čeksterytè, G. Dabkevičienè, B. Kurtinaitienè, and R. Navakauskiené, "Comparative Proteomic Analysis of Pollen of Trifolium Pratense, T. Alexandrinum and T. Repens," Zemdirbyste-Agriculture, vol. 101, no. 4, pp. 453-460, Dec. 2014. https://doi.org/10.13080/z-a.2014.101.058

[5] D. Matuzevičius, E. Žurauskas, R. Navakauskienė, and D. Navakauskas, "Improved Proteomic Characterization of Human Myocardium and Heart Conduction System by Computational Methods," Biologija, vol. 54, no. 4, pp. 283-289, Jan. 2008. https://doi.org/10.2478/v10054-008-0058-9

[6] R. Navakauskiene, S. Baronaite, D. Matuzevicius, I. Zaikova, A. Arlauskiene, D. Navakauskas, and G. Treigyte, "Identification and Characterization of Amniotic Fluid Proteins Incident to Normal, Preeclampsia and Polyhydramnios Pregnancies," Current Proteomics, vol. 13, no. 3, pp. 206-217, Sep. 2016. https://doi.org/10.2174/1570164613666160531113559

[7] A Guide to Polyacrylamide Gel Electrophoresis and Detection, Bio-Rad Labaratories, Inc., Bulletin 6040, 2013.

[8] A. Serackis and D. Navakauskas, "Treatment of Over-Saturated Protein Spots in Two-Dimensional Electrophoresis Gel Images," Informatica, vol. 21, no. 3, pp. 409-424, Nov. 2010.

[9] D. Matuzevičius, A. Serackis, and D. Navakauskas, "Mathematical model of oversaturated protein spots," Elektronika ir Elektrotechnika, vol. 73, no. 1, pp. 63-68, Jan. 2007.

[10] P. Kovesi, "Image Features From Phase Congruency," Videre: J. Computervis. Res., vol. 1, no. 3, pp. 1-26, 1999.

[11] P. Kovesi, "Symmetry and Asymmetry From Local Phase," in Tenth Australian joint conference on artificial intelligence, 1997, pp. 2-4.

[12] M. Natale, B. Maresca, P. Abrescia, and E. M. Bucci, "Image Analysis Workflow for 2-D Electrophoresis Gels Based on ImageJ," Proteomics Insights, vol. 4, p. PRI.S7971, Jan. 2011.

http://doi.org/10.4137/PRI.S7971 
[13] A. W. Dowsey, M. J. Dunn, and G.-Z. Yang, "The Role of Bioinformatics in Two-Dimensional Gel Electrophoresis," Proteomics, vol. 3, no. 8, pp. 1567-1596, Aug. 2003. https://doi.org/10.1002/pmic.200300459

[14] C. V. Cannistraci, F. M. Montevecchi, and M. Alessio, "MedianModified Wiener Filter Provides Efficient Denoising, Preserving Spot Edge and Morphology in 2-DE Image Processing," Proteomics, vol. 9, no. 21, pp. 4908-4919, Nov. 2009. https://doi.org/10.1002/pmic.200800538

[15] T. S. Lee, "Image Representation Using 2D Gabor Wavelets," IEEE Transactions on Pattern Analysis and Machine Intelligence, vol. 18, no. 10, pp. 959-971, 1996. https://doi.org/10.1109/34.541406

[16] K. N. Kay, T. Naselaris, R. J. Prenger, and J. L. Gallant, "Identifying Natural Images From Human Brain Activity," Nature, vol. 452, no. 7185 , pp. 352-355, Mar. 2008. https://doi.org/10.1038/nature06713

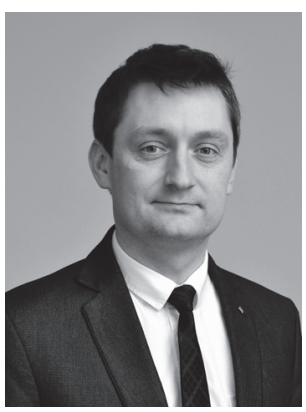

Artūras Serackis is a Professor at the Department of Electronic Systems at VGTU. He received his B. sc. degree in Electronic Engineering in 2002, M. sc. in Electronic Engineering in 2004 and his Doctor of Electrical and Electronic Engineering degree in 2008 at VGTU. In 2012, he received the title of Associate Professor and in 2017, the title of Professor at VGTU.

He was a member of the organizing committees at various international conferences, such as NORCHIP (2013), EMD (2012), ICDIPC (2012), MIKON (2010), SPS (2010). His main research interests include real-time image and signal processing, intelligent systems.

He has published more than 50 papers in reviewed journals and conference proceedings, a monograph in the field of intelligent electronic systems and two textbooks.

Mr. Serackis has successfully completed nine research and development projects, currently supervising four PhD students. He is a Senior Member of the IEEE and Chair of IEEE Lithuania Section Signal Processing Society/Computational Intelligence Society/Communication Society joint chapter.

Address: Vilnius Gediminas Technical University, Faculty of Electronics, Naugarduko str. 41 - 413, Vilnius, LT-03227, Lithuania.

E-mail: arturas.serackis@vgtu.lt

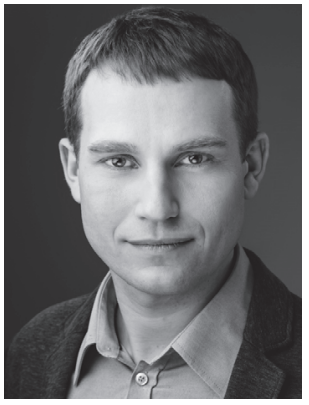

Dalius Matuzevičius obtained his B. sc. and M. sc. degrees in Electronic Engineering and a $\mathrm{PhD}$ degree in Electrical and Electronic engineering from Vilnius Gediminas Technical University in 2004, 2006, and 2010, respectively. In 2015 , he received the title of Associate Professor at VGTU.

$\mathrm{He}$ is currently a Professor at the Department of Electronic Systems of Vilnius Gediminas Technical University. His main professional interests are machine and biological vision, machine learning, bioinformatics.

Mr. Matuzevičius is a Senior Member of the

IEEE.

Address: Vilnius Gediminas Technical University, Faculty of Electronics, Naugarduko str. 41 - 413, Vilnius, LT-03227, Lithuania.

E-mail: dalius.matuzevicius@vgtu.lt

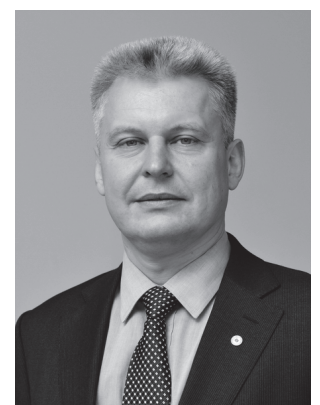

Dalius Navakauskas is a Professor at the Department of Electronic Systems at Vilnius Gediminas Technical University, Lithuania. He received his B. sc. diploma (with honours) in Radio-Electronics Engineering in 1992, M. sc. in Electronics in 1994, Doctor of Electrical and Electronic Engineering degree in 1999, passed the habilitation procedure in Informatics Engineering in 2005, received the title of Professor in 2008, all at Vilnius Gediminas Technical University.
His main research interests include computational intelligence, signal and image processing, bioinformatics.

Professor Navakauskas is a Senior Member of the IEEE, an active member of IEEE Computational Intelligence and Signal Processing Societies, and currently serves as Vice-Chair of IEEE Lithuania Section.

He has published more than 75 internationally reviewed papers, a monograph in the field of intelligent electronic systems and four textbooks for students. Address: Vilnius Gediminas Technical University, Faculty of Electronics, Naugarduko str. 41 - 426, Vilnius, LT-03227, Lithuania.

E-mail: dalius.navakauskas@vgtu.lt

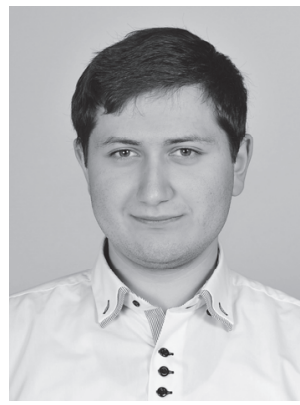

Eldar Šabanovič obtained his B. sc. and M. sc. degrees in Electronic Engineering from Vilnius Gediminas Technical University in 2013 and 2015, respectively. He is a third-year doctorate student in the field of electrical and electronic engineering.

Currently, he works as Lecturer and Junior Researcher at Vilnius Gediminas Technical University, Vilnius, Lithuania. His research interests include computer vision, artificial neural networks and image analysis.

Mr. Šabanovič is a member of IEEE, a member of IEEE Computer, Computational Intelligence, Signal Processing Society, Aerospace and Electronic Systems Societies, and currently serves as Student Representative of IEEE Lithuania Section.

Address: Vilnius Gediminas Technical University, Faculty of Electronics, Naugarduko str. 41 - 415, Vilnius, LT-03227, Lithuania.

E-mail: eldar.sabanovic@vgtu.lt

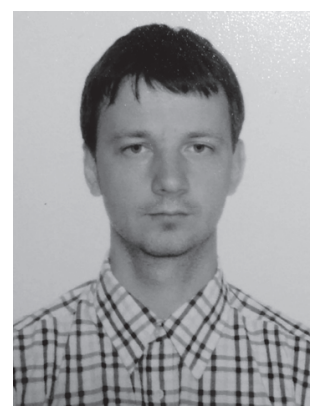

Andrius Katkevičius obtained his B. sc., M. sc. and Ph.D. degrees in Electrical and Electronic Engineering from Vilnius Gediminas Technical University in 2007, 2009, and 2013, respectively.

$\mathrm{He}$ is an Associate Professor at the Department of Electronic Systems of Vilnius Gediminas Technical University. His main research interests include the electromagnetic field theory, super-high frequency technologies and microwave devices, signal processing, multimedia and embedded systems.

Mr. Katkevičius is a member of the IEEE and an active member of the IEEE Microwave Theory and Techniques.

Address: Vilnius Gediminas Technical University, Faculty of Electronics, Naugarduko str. 41 - 415, Vilnius, LT-03227, Lithuania.

E-mail: andrius.katkevicius@vgtu.lt

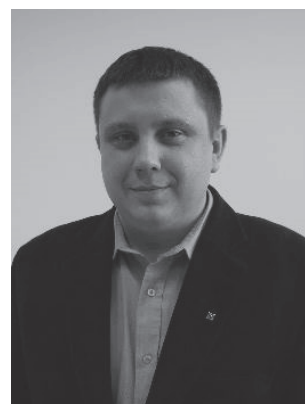

Darius Plonis received his B. sc., M. sc. and Ph.D. degrees in Electrical and Electronic Engineering from Vilnius Gediminas Technical University in 2008, 2010, and 2014, respectively.

$\mathrm{He}$ is an Associate Professor at the Department of Electronic Systems of Vilnius Gediminas Technical University. His main research interests include electromagnetic field theory, super-high frequency technologies and microwave devices, signal processing, multimedia and embedded systems.

Mr. Plonis is a member of the IEEE, an active member of IEEE Microwave Theory and Techniques, and currently serves as Secretary of IEEE Lithuania Section.

Address: Vilnius Gediminas Technical University, Faculty of Electronics, Naugarduko str. 41 - 413, Vilnius, LT-03227, Lithuania.

E-mail: darius.plonis@vgtu.lt 